\title{
NANETS/SNMMI Procedure Standard for Somatostatin Receptor-Based Peptide Receptor Radionuclide Therapy with ${ }^{177}$ Lu-DOTATATE
}

\author{
Thomas A. Hope ${ }^{1,2}$, Amanda Abbott ${ }^{3}$, Karen Colucci ${ }^{4}$, David L. Bushnell ${ }^{5,6}$, Linda Gardner ${ }^{7}$, William S. Graham ${ }^{1}$, \\ Sheila Lindsay ${ }^{8}$, David C. Metz ${ }^{9}$, Daniel A. Pryma ${ }^{10}$, Michael G. Stabin ${ }^{11}$, and Jonathan R. Strosberg ${ }^{12}$ \\ ${ }^{I}$ Department of Radiology and Biomedical Imaging, University of California, San Francisco, San Francisco, California; ${ }^{2}$ Department \\ of Radiology, San Francisco VA Medical Center, San Francisco, California; ${ }^{3}$ Dana-Farber Cancer Institute, Boston, \\ Massachusetts; ${ }^{4}$ Lehigh Valley Health Network, Allentown, Pennsylvania; ${ }^{5}$ Department of Radiology, Carver College of Medicine, \\ University of Iowa, Iowa City, Iowa; ${ }^{6}$ Iowa City VA Medical Center, Iowa City, Iowa; ${ }^{7}$ Department of Nuclear Medicine, University \\ of California, Los Angeles, Los Angeles, California; ${ }^{8}$ Division of Hematology/Oncology, Department of Medicine, University of \\ California, San Francisco, San Francisco, California; ${ }^{9}$ Division of Gastroenterology, Department of Medicine, Perelman School \\ of Medicine at the University of Pennsylvania, Philadelphia, Pennsylvania; ${ }^{10}$ Division of Nuclear Medicine \& Clinical \\ Molecular Imaging, Department of Radiology, Perelman School of Medicine at the University of Pennsylvania, Philadelphia, \\ Pennsylvania; ${ }^{11}$ NV5/Dade Moeller, Richland, Washington; and ${ }^{12}$ Department of Gastrointestinal Oncology, Moffitt Cancer \\ Center, Tampa, Florida
}

With the recent approval of ${ }^{177} \mathrm{Lu}$-DOTATATE for use in gastroenteropancreatic neuroendocrine tumors, access to peptide receptor radionuclide therapy is increasing. Representatives from the North American Neuroendocrine Tumor Society and the Society of Nuclear Medicine and Molecular Imaging collaborated to develop a practical consensus guideline for the administration of ${ }^{177} \mathrm{Lu}-$ DOTATATE. In this paper, we discuss patient screening, maintenance somatostatin analog therapy requirements, treatment location and room preparation, drug administration, and patient release as well as strategies for radiation safety, toxicity monitoring, management of potential complications, and follow-up. Controversies regarding the role of radiation dosimetry are discussed as well. This document is designed to provide practical guidance on how to safely treat patients with this therapy.

J Nucl Med 2019; 60:937-943

DOI: 10.2967/jnumed.118.230607

\section{PREAMBLE}

The present guidelines/standards were developed collaboratively by the North American Neuroendocrine Tumor Society (NANETS) and the Society of Nuclear Medicine and Molecular Imaging (SNMMI). NANETS is a multidisciplinary professional society of neuroendocrine specialists in North America that was founded in 2005. NANETS's mission is to improve neuroendocrine tumor disease management through increased research and educational opportunities. NANETS is committed to a multidisciplinary approach and consists of doctors and scientists involved in different specialties of neuroendocrine tumors (NETs). The SNMMI is an international

For correspondence or reprints contact: Thomas A. Hope, University of California, San Francisco, Department of Radiology and Biomedical Imaging, 505 Parnassus Ave., San Francisco, CA 94143.

Email: thomas.hope@ucsf.edu

COPYRIGHT (C) 2019 by the Society of Nuclear Medicine and Molecular Imaging. scientific and professional organization founded in 1954 to promote the science, technology, and practical application of nuclear medicine. In addition to publishing journals, newsletters, and books, the SNMMI also sponsors international meetings and workshops designed to increase the competencies of nuclear medicine practitioners and to promote new advances in the science of nuclear medicine.

This collaboration between NANETS and SNMMI aims to define new procedure standards for peptide receptor radionuclide therapy (PRRT) to improve the quality of service to patients. Existing practice guidelines will be reviewed for revision or renewal as appropriate, on their fifth anniversary or sooner, if indicated. Each practice standard has undergone a thorough consensus process in which it has been subjected to extensive review. Much of the content within the guidelines are based on working group experience. These procedure standards are intended to assist practitioners in providing appropriate nuclear medicine care for patients. They are not inflexible rules or requirements of practice and are not intended, nor should they be used, to establish a legal standard of care. For these reasons and those set forth below, NANETS and SNMMI caution against the use of these guidelines in litigation in which the clinical decisions of a practitioner are called into question. The ultimate judgment regarding the propriety of any specific procedure or course of action must be made by medical professionals taking into account the unique circumstances of each case. Thus, there is no implication that an approach differing from the guidelines, standing alone, is below the standard of care. To the contrary, a conscientious practitioner may responsibly adopt a course of action different from that set forth in the guidelines when, in the reasonable judgment of the practitioner, such course of action is indicated by the condition of the patient, limitations of available resources, or advances in knowledge or technology subsequent to publication of the guidelines.

The variety and complexity of human conditions make it impossible to always reach the most appropriate diagnosis or to predict with certainty a particular response to treatment. Therefore, it should be recognized that adherence to these guidelines will not 
ensure an accurate diagnosis or a successful outcome. All that should be expected is that the practitioner will follow a reasonable course of action based on current knowledge, available resources, and the needs of the patient to deliver effective and safe medical care. The sole purpose of these procedure standards is to assist practitioners in achieving this objective.

\section{BACKGROUND}

NETs are a heterogeneous group of malignancies that frequently overexpress somatostatin receptors (SSTRs) (1). NETs can be imaged using somatostatin analogs (SSAs) labeled with ${ }^{68} \mathrm{Ga}\left({ }^{68} \mathrm{Ga}-\right.$ DOTATATE and ${ }^{68} \mathrm{Ga}$-DOTATOC) (2). $\beta$-emitting radionuclides such as ${ }^{177} \mathrm{Lu}$ can be used for PRRT (3). The NETTER-1 trial demonstrated prolonged progression-free survival in midgut NET patients treated with 4 cycles of ${ }^{177}$ Lu-DOTATATE, which subsequently led to the approval of this therapy (4).

\section{TREATMENT OVERVIEW}

${ }^{177} \mathrm{Lu}$-DOTATATE is administered at an activity of $7.4 \mathrm{GBq}$ (200 mCi) every $8 \pm 1 \mathrm{wk}$ for 4 cycles. Combined with prophylactic amino acid infusions and antiemetics, each treatment visit can last approximately 5-8 h (Fig. 1). Before starting PRRT treatments, each treatment site must ensure that ${ }^{177} \mathrm{Lu}$ is included in their institutional radioactive materials license. A detailed review of procedures surrounding ${ }^{177} \mathrm{Lu}$-DOTATATE therapy is provided below.

\section{PATIENT SCREENING}

Patients should be evaluated by a multidisciplinary NET team, including a cancer specialist with expertise in the medical management of NETs as well as a nuclear medicine physician or appropriate authorized user to decide on the appropriateness and timing of PRRT in individual patients. Potential candidates should undergo an SSTR PET scan or SSTR scintigraphy ( ${ }^{111}$ In-pentetreotide) to demonstrate adequate SSTR expression (2). Traditionally, SSTR expression on ${ }^{111}$ In-pentetreotide greater than background hepatic uptake has been considered an eligibility requirement for PRRT (5). Necessary levels of SSTR expression on ${ }^{68} \mathrm{Ga}$-based SSTR PET have not been clearly defined, but lesion uptake should exceed background hepatic uptake. Laboratory values should be checked shortly before the treatment is ordered (typically $2 \mathrm{wk}$ before each cycle). These should include blood urea nitrogen, creatinine, albumin, alkaline phosphatase, aspartate aminotransferase, alanine aminotransferase, total bilirubin, white blood cell with differential, hemoglobin, and platelet counts. The threshold values provided in Table 1 should be taken as general eligibility guidelines for therapy.

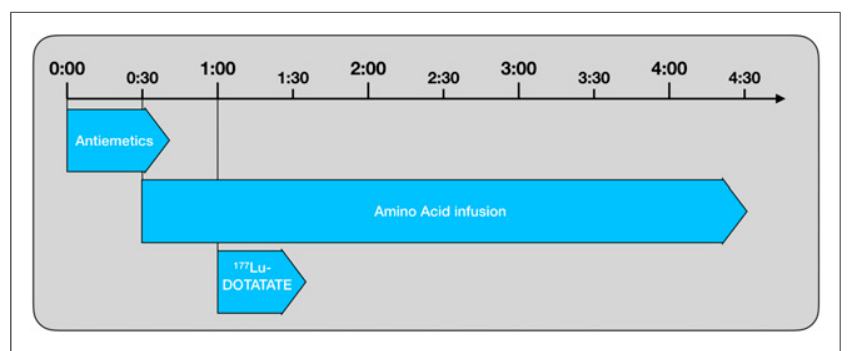

FIGURE 1. Timeline of administration of antiemetics, amino acids, and ${ }^{177}$ Lu-DOTATATE during PRRT. Antiemetics can be repeated during amino acid infusion as needed.

\section{SOMATOSTATIN ANALOG THERAPY}

SSA therapy is used frequently to treat NET patients, and SSAs are typically administered in depot formulations every $4 \mathrm{wk}$. It is recommended that PRRT treatments be scheduled at least $4 \mathrm{wk}$ after the last long-acting SSA therapy to prevent interference with SSTR binding. For symptomatic patients, short-acting SSAs being used as a bridge should be stopped at least $24 \mathrm{~h}$ before treatment. Subsequent SSA doses can be administered as soon as several hours after the completion of the radiopharmaceutical therapy. During and after completion of PRRT, it is generally agreed that syndromic patients should remain on SSA therapy. It is unclear whether patients with nonfunctional tumors should remain on SSA treatment regardless of whether or not they had progressed on SSA therapy before initiation of PRRT. In the NETTER-1 study, all patients remained on long-acting release octreotide despite prior progression on this drug, and the ${ }^{177}$ Lu-DOTATATE package insert suggests that patients should remain on SSAs for up to 18 mo after treatment (6). However, there are no clear data to support or refute this recommendation.

\section{TREATMENT LOCATION}

Sites have the option to provide PRRT in an inpatient or outpatient setting, within the oncology infusion clinic or nuclear medicine department, or a combination of both locations. Most sites in the United States treat in the outpatient setting. Oncology nursing staff are often more accustomed to the complexities of required concomitant medication infusions and patient monitoring than nuclear medicine staff, but nuclear medicine staff are well trained in radiation safety and the necessary precautions required during administration of radioactivity. If a combination approach is taken (i.e., the patient is transported between departments for specific components of the procedure), extra caution regarding radiation protection is required for patient travel. When treated as an outpatient, a patient should be forewarned of the uncommon possibility of an overnight hospital stay should a complication such as a neuroendocrine hormonal crisis or severe emesis occur (6).

\section{ROOM PREPARATION}

Because body fluids (primarily urine) are radioactive after ${ }^{177} \mathrm{Lu}-$ DOTATATE administration, room preparation is essential to reduce potential contamination. For example, patient stretchers, chairs, floors, and lower walls can be covered with a prophylactic protective covering (Fig. 2). Furthermore, whereas a treatment suite with an

TABLE 1

Recommended Laboratory Thresholds for PRRT Treatment

\begin{tabular}{ll}
\hline \multicolumn{1}{c}{ Laboratory } & $\begin{array}{c}\text { Acceptable value before } \\
\text { first treatment }\end{array}$ \\
\hline Hemoglobin (HGB) & $>8 \mathrm{~g} / \mathrm{dL}$ \\
\hline White blood cell count (WBC) & $>2 \mathrm{~K} / \mathrm{mm}^{3}$ \\
Platelet count (PLT) & $>70 \mathrm{~K} / \mathrm{mm}^{3}$ \\
Estimated glomerular filtration & $>50 \mathrm{~mL} / \mathrm{min}^{3}$ \\
$\quad$ rate (eGFR) & $\leq 3 \times \mathrm{ULN}$ \\
Total bilirubin & $>3.0 \mathrm{~g} / \mathrm{dL}$ \\
Serum albumin &
\end{tabular}

ULN $=$ upper limits of normal. 


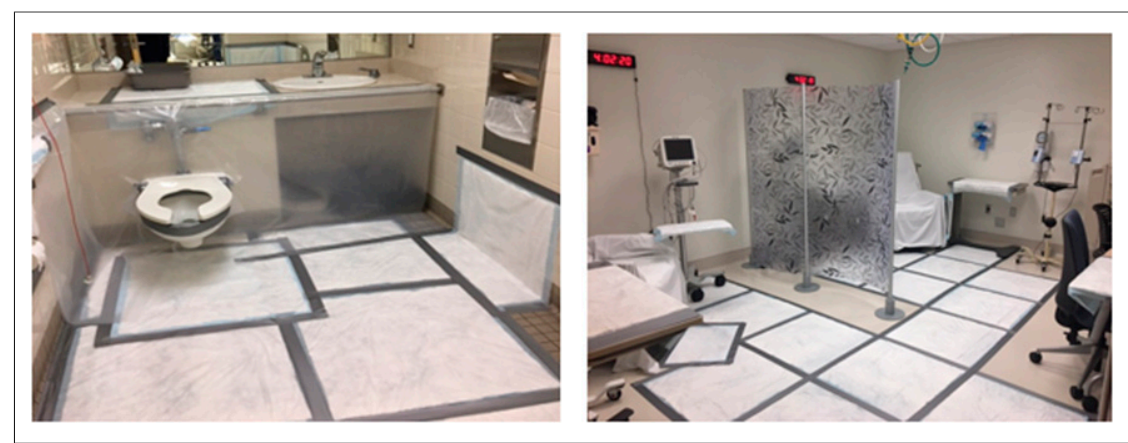

FIGURE 2. Example room preparation for therapy. A dedicated bathroom should be used and wrapped in order to prevent urine contamination.

attached toilet is ideal, having a dedicated toilet nearby is acceptable as the patient will need to void frequently on the completion of the ${ }^{177} \mathrm{Lu}$-DOTATATE infusion and may need assistance. Local rules related to radioactive waste materials should be followed under the guidance of a local radiation safety officer.

\section{PATIENT PREPARATION}

It may be helpful to have patients change into hospital scrubs or gowns on arrival in order to avoid potential contamination of personal belongings. If stress urinary incontinence is a concern, disposable undergarments are also recommended. For some patients, a Foley catheter with acrylic shielding of the Foley bag may be necessary. However, routine bladder catheterization is not recommended.

A peripheral vein in the antecubital fossa is the preferred location for venous access, and intravenous lines in both arms are preferred, one to administer the radioactivity and one to administer the amino acid solution. If 2 lines are not possible, the amino acids and radiotherapy may be infused through the same line. Central lines may be used for the administration of the premedications and the amino acid solution in the case of patients with difficult peripheral venous access; however, a peripheral line is the recommended route for the administration of the ${ }^{177} \mathrm{Lu}$-DOTATATE infusion, as the use of a central line has not been studied. Intermittent assessment of ongoing vascular access is necessary throughout the procedure due to the high osmolarity of the amino acid solutions, and local reactions at the infusion site may occur. Additionally, patency of the peripheral intravenous site should be monitored continuously throughout the infusion of the ${ }^{177} \mathrm{Lu}$-DOTATATE.

\section{AMINO ACID SOLUTIONS}

Administration of an amino acid solution with the appropriate lysine and arginine concentration (Table 2) before, during, and after the ${ }^{177} \mathrm{Lu}$-DOTATATE infusion is required to decrease reabsorption of ${ }^{177} \mathrm{Lu}$-DOTATATE via the proximal renal tubules and thereby decrease the radiation dose to the kidneys (7). Several highconcentration commercial amino acid solutions are currently available with the correct concentration of arginine and lysine (Table 3); however, they also include additional amino acids, which raise the osmolality of the solution and are associated with significant nausea and vomiting during infusion. The target infusion rate of the commercial amino acid solutions should reach $320 \mathrm{~mL} / \mathrm{h} .{ }^{177} \mathrm{Lu}-\mathrm{DOTA}-$ TATE should generally not be administered until this rate is reached or until one eighth of the total volume of amino acid solution has been infused. The amino acid solution should infuse concurrently with the ${ }^{177} \mathrm{Lu}$-DOTATATE, and continue at $320 \mathrm{~mL} / \mathrm{h}$ or greater until the total volume has been administered. Commencing at a low rate of $100 \mathrm{~mL} / \mathrm{h}$ for the highconcentration amino acid solution and increasing slowly (e.g., by $20-50 \mathrm{~mL} / \mathrm{h}$ every 15-20 min) has been somewhat successful at reducing side effects relating to nausea or vomiting.

An alternative compounded amino acid formulation consisting solely of $25 \mathrm{~g}$ of lysine and $25 \mathrm{~g}$ of arginine diluted in $1 \mathrm{~L}$ of normal saline for injection should be considered (8). The compounded 2-amino-acid solution is substantially less emetogenic and can generally be infused over a shorter period of time. The use of arginine-lysine formulations may be preferred due to improved tolerability; however, due to licensing requirements and compounding regulations, compounded argininelysine formulations may not be available at many institutions. When using the compounded arginine-lysine solution, it should be infused at a rate of $250 \mathrm{~mL} / \mathrm{h}$ for $4 \mathrm{~h}$, commencing $30 \mathrm{~min}$ before the treatment with the radiopharmaceutical.

\section{ANTIEMETIC MEDICATIONS}

Nausea and vomiting are common during the administration of commercial amino acid solutions when infusion rates are above $250 \mathrm{~mL} / \mathrm{h}$. Therefore, it is recommended to use an intravenous premedication regime consisting of a 5-HT3 antagonist (e.g., granisetron, ondansetron, or palonosetron), an NK1 receptor antagonist (e.g., fosaprepitant), and an $\mathrm{H} 2$ receptor antagonist (e.g., famotidine). When increasing the amino acid rate to the target of $320 \mathrm{~mL} / \mathrm{h}$, additional doses of the 5-HT3 antagonist may be required with the addition of a D2 receptor antagonist (e.g., prochlorperazine). Benzodiazepines may also be required for anticipatory nausea and vomiting (9). Steroids, such as dexamethasone, can also be administered after infusion of ${ }^{177} \mathrm{Lu}$-DOTATATE.

Additionally, cooling and pressure aids may also be beneficial to help with the possible side effects of the amino acid solution infusion. Patient education is highly important at the beginning of the procedure to ensure the patient understands the importance of where and how to contain emesis under these circumstances.

Patients receiving compounded arginine-lysine generally require only a 5-HT3 antagonist as prophylactic medication.

\section{RADIOPHARMACEUTICAL ADMINISTRATION}

Treatment centers can choose among different infusion methods for ${ }^{177} \mathrm{Lu}$-DOTATATE administration, including the gravity method,

TABLE 2

Content Requirements for the Amino Acid Solution

\begin{tabular}{ll}
\hline \multicolumn{1}{c}{ Item } & \multicolumn{1}{c}{ Specification } \\
\hline Lysine $\mathrm{HCl}$ content & Between 18 and $24 \mathrm{~g}$ \\
Arginine $\mathrm{HCl}$ content & Between 18 and $24 \mathrm{~g}$ \\
Volume & 1.5 to $2.2 \mathrm{~L}$ for commercial \\
& $(1.0 \mathrm{~L}$ for compounded $)$ \\
Osmolarity & $<1,050$ mOsmol \\
\hline
\end{tabular}


TABLE 3

Currently Available Commercial Amino Acid Solutions

\begin{tabular}{lll}
\hline \multicolumn{1}{c}{ Formulation } & Amino acid concentration & Dilution \\
\hline Aminosyn II $10 \%$ & $21 \mathrm{~g}$ of lysine, $20.4 \mathrm{~g}$ of arginine in $2 \mathrm{~L}$ & Dilute to approximately $2 \mathrm{~L}$ \\
\hline Aminosyn II $15 \%$ & $23.6 \mathrm{~g}$ of lysine, $22.9 \mathrm{~g}$ of arginine in $1.5 \mathrm{~L}$ & Dilute to approximately $2.2 \mathrm{~L}$ \\
\hline Clinisol $15 \%$ & $18 \mathrm{~g}$ of lysine, $18 \mathrm{~g}$ of arginine in $1.6 \mathrm{~L}$ & Dilute to approximately $2.1 \mathrm{~L}$ \\
\hline Plenamine $15 \%$ & $18.8 \mathrm{~g}$ of lysine, $23.5 \mathrm{~g}$ of arginine in $1.6 \mathrm{~L}$ & Consult* $^{\star}$ \\
\hline Trophamine $10 \%$ & $18 \mathrm{~g}$ of lysine, $26 \mathrm{~g}$ of arginine in $2.2 \mathrm{~L}$ & Consult* $^{\star}$
\end{tabular}

${ }^{*}$ Consult with treating physician as lysine-arginine concentration may be outside specifications, which may increase adverse events.

the saline infusion method, the pump method with a vial, and the pump method with a syringe (Fig. 3) (6). Supplemental Table 1 (supplemental materials are available at http://jnm.snmjournals.org) provides step-by-step instructions for each method (10). The saline infusion method may result in leakage from the vial, and care should be taken to prevent contamination. The pump method with a syringe can result in extra radiation exposure to the technologists when drawing the activity from the vial and higher residual values. The pump method with the vial requires the availability of an automated infusion pump. Regardless of the method used, one should use appropriate radiation shielding and aseptic technique when preparing and administering the radiopeptide solution, including wearing appropriate personal protective equipment, using tongs when handling the vial to minimize radiation exposure, confirming the amount of the radioactivity of the radiopeptide vial (and syringe if applicable) with an appropriate dose calibrator before administration, and inspecting the product visually for particulate matter and discoloration under a shielded screen (the vial should not be used if particulates or discoloration are present). The therapy dosage should be administered over 30 min and should not be administered as a bolus.

\section{DOSAGE MODIFICATIONS FOR ADVERSE REACTIONS}

In patients who have baseline renal, liver, or bone marrow dysfunction, or in those who develop toxicity while on treatment, modification of the administered ${ }^{177} \mathrm{Lu}$-DOTATATE activity can be considered (6). In patients with preexisting toxicity, decreased administered activity or longer intervals between administrations can be considered. Bone marrow toxicity is the most common adverse event to develop during treatment. In patients experiencing myelosuppression greater than grade 1 , one can delay treatment, allowing bone marrow function to recover, administer a lower administered activity (i.e., $3.7 \mathrm{GBq}[100 \mathrm{mCi}]$ ) during the next treatment, or permanently stop therapy. Oftentimes, thrombocytopenia will resolve with a delay. Renal and liver toxicity rarely occur during treatment, but if patients develop toxicity that is attributable to ${ }^{177}$ Lu-DOTATATE (e.g., elevated bilirubin or reduction in kidney function), therapy should be withheld until toxicity resolves. Because of issues related to differential reimbursement based on the administered activity, it may be difficult to administer at half the normal activity. Therefore, it may only be feasible to prolong the delay between treatments.

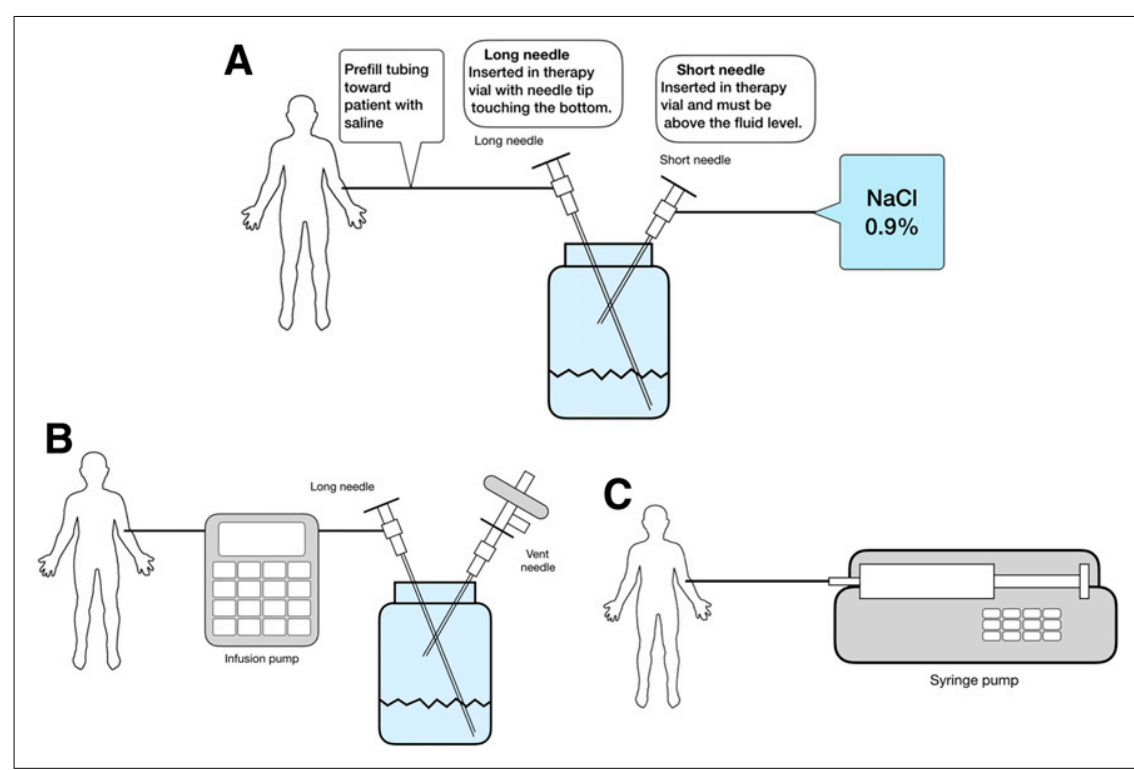

FIGURE 3. Administration techniques. (A) Gravity method. (B) Pump method with vial. (C) Pump method with syringe. Please see Supplemental Table 2 for further details on the administration techniques.

\section{PATIENT MONITORING AND POTENTIAL REACTIONS}

\section{Hormonal Crisis (Carcinoid Crisis)}

Neuroendocrine hormonal crises due to excessive release of hormones or bioactive substances develop in $1 \%$ of patients and typically occur during treatment or within $2 \mathrm{~d}$ after the initial treatment $(6,11)$. Typical clinical manifestations include cutaneous flushing, diarrhea, bronchospasm, and hypertension. Hormonal crises can be treated with intravenous high-dose SSAs, intravenous fluids, corticosteroids, and correcting of electrolyte disturbances in patients with diarrhea or vomiting. Pretreating patients at high-risk for crisis has been suggested, although this is not done at most centers (12).

\section{Infiltration of ${ }^{177}$ Lu-DOTATATE}

Prevention of infiltration is critical and includes testing the intravenous line patency before administration of the radiopharmaceutical, direct observation of the site during 
the administration, and rapid intervention if swelling or pain develop. If infiltration occurs, clearance of the radiotracer from the site can be facilitated with warm packs, compression, and elevation (13). Infiltration must be reported to radiation safety for monitoring and calculation of skin dose.

\section{RADIATION SAFETY}

\section{Contamination}

With a half-life of $6.6 \mathrm{~d},{ }^{177} \mathrm{Lu}$ raises the possibility of prolonged contamination. Blood and urine are the main sources of contamination during and after radionuclide administration. Because ${ }^{177} \mathrm{Lu}$-DOTATATE is primarily excreted in urine, with a cumulative excretion of $44 \%$ within $5 \mathrm{~h}, 58 \%$ within $24 \mathrm{~h}$, and $65 \%$ within $48 \mathrm{~h}$ after administration, the main focus for individual patients for the first $3 \mathrm{~d}$ after therapy is on preventing urinary contamination (6). Of note, when produced by neutron activation, there is a long-lived ${ }^{177} \mathrm{Lu}$ contaminant, ${ }^{177 \mathrm{~m}} \mathrm{Lu}$, which has a half-life of $160 \mathrm{~d}$ and needs to be surveyed for before disposal of radioactive waste from the treatment center. This issue is of greater importance in locations that treat patients in an inpatient setting. Emesis can contain small amounts of radioactive material and should be treated as contaminated and disposed of appropriately.

\section{Preparation for Inpatient Therapy}

For patients who require an overnight stay, typically due to medical complications, the recommendations largely follow those already in place for ${ }^{131} \mathrm{I}$ inpatients, noting that the external dose rate from ${ }^{177} \mathrm{Lu}$ is significantly lower than ${ }^{131} \mathrm{I}$ given the lower energy and abundance of its $\gamma$-emissions. Nursing personnel will need to be instructed in pertinent radiation safety precautions (i.e., potential for contamination related primarily to the patient's blood and urine), but also be advised that nausea and vomiting related to the administration of the amino acid solution may occur. Universal precautions (e.g., gloves, gowns, shoe covers) should be used to avoid contact with patient bodily fluids. If blood or urine specimens are needed for laboratory testing, nursing staff should be advised to collect the smallest amount necessary for testing. Nursing staff should be provided with radiation monitoring devices (passive dosimeter, direct-reading dosimeter). Ideally, radiation safety staff should help prepare the patient's bed, floor, and bathroom to minimize potential radioactive contamination from patient bodily fluids. Institutional radiation safety guidelines should be developed for general nursing care of ${ }^{177} \mathrm{Lu}$-DOTATATE inpatients including approaches to medical emergencies or patient deaths before a ${ }^{177} \mathrm{Lu}$-DOTATATE therapy program is established.

\section{Release Criteria}

To ensure that radiation dose to members of the public remains less than $5 \mathrm{mSv}$ (500 mrem), the patient should be provided with instructions (Table 4) at discharge. Each center should determine its own recommendations based on each patient's specific circumstances and local regulations, but we have provided a basic guidance in Table 4 for minimizing exposure to others and potential urine contamination (14). The average exposure at $1 \mathrm{~m}$ immediately after treatment is $1.8 \pm 0.5 \mathrm{mrem} / \mathrm{h}$ and at the time of discharge is $0.9 \pm 0.4 \mathrm{mrem} / \mathrm{h}$ (combined institutional experiences from more than 100 therapies). This exposure is less than that from ${ }^{131}$ I therapy and below release criteria from published Nuclear Regulatory Commission guidelines (15). The time periods for following various instructions will vary, but $3 \mathrm{~d}$ should be sufficient for resumption of most public activities, given the physical half-life of $6.7 \mathrm{~d}$, the mean effective blood elimination half-life of $3.5 \pm 1.4 \mathrm{~h}$, and the mean terminal blood half-life of $71 \pm 28 \mathrm{~h}(6)$. Extra precautions should be taken to minimize exposure of young children and pregnant women. The mathematics of release criteria for any nuclear medicine therapy patients have been addressed comprehensively (14). The RADAR website has an online tool that allows for calculation of cumulative doses to family members or members of the public from exposure to patients treated with ${ }^{177} \mathrm{Lu}$-DOTATATE (16).

\section{Pregnancy}

Radiopharmaceutical therapy is almost universally contraindicated during pregnancy. Therapy must be delayed until childbirth or termination of pregnancy. Breast feeding should be stopped for treatment and not be restarted until $2.5 \mathrm{mo}$ after the final therapy. Future children may be breastfed. Contraception should be used for 6 mo after completion of the final treatment.

\section{Issues with Cremation and Patient Death}

It is important to notify the local radiation safety officer of a death involving a radioactive patient. Deceased patients should be appropriately labeled, and the death certificate should note that the patient is radioactive. If possible the radiation safety officer should appropriately train the medical examiners and the mortuary personnel, as well as perform radiation surveys. Please refer to the National Council on Radiation Protection and Measurements (NCRP) Report no. 161 (NCRP 2008) for additional guidance on the management of radiation accident victims, regarding guidelines for the medical examiner and mortuary personnel (17).

\section{DOSIMETRY AND POSTTREATMENT IMAGING}

Although dosimetry was not a part of the phase III NETTER-1 trial, there may be a role for patient-specific dosimetry when considering cumulative renal and bone marrow dose. There is a large variability in tumor and organ uptake of radiolabeled SSAs across patients, which suggests that tailored dosimetry may be useful for

TABLE 4

Radiation Safety Recommendations After ${ }^{177}$ Lu-DOTATATE Treatment

\begin{tabular}{ll}
\hline Duration & \multicolumn{1}{c}{ Recommendations } \\
\hline $3 \mathrm{~d} \quad$ Sleep & $\begin{array}{l}\text { Sleep in separate bed, avoid intimate contact. For } \\
\text { infants/children or pregnant partner, the time } \\
\text { period should be extended. }\end{array}$ \\
& Urination \\
& Flush toilet twice with the lid closed after each use \\
& (all patients should be advised to sit down when \\
& urinating to minimize/avoid splashing), and to \\
& use separate towels and washcloths. \\
& General recommendations \\
& Use a general distance guideline of no closer than \\
& 3 feet for not more than $1 \mathrm{~h}$ per day. Try to \\
maintain a distance of 6 feet from others. \\
Minimize public transportation and use of public \\
facilities. \\
Return to work in 3 d, depending on patient \\
tolerance.
\end{tabular}




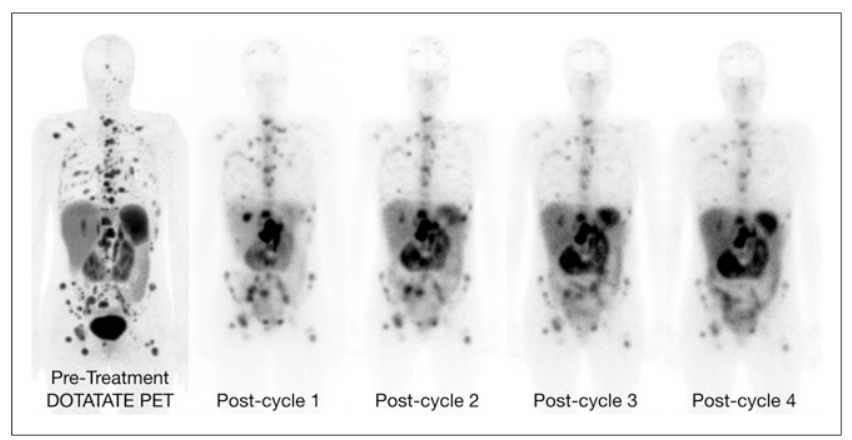

FIGURE 4. Whole-body images acquired after administration of ${ }^{177} \mathrm{Lu}-$ DOTATATE in different therapy cycles.

${ }^{177} \mathrm{Lu}$-DOTATATE therapy (18). For example, 1 study showed a biologically effective dose to the kidneys ranging from 9 to more than 40 Gy (19). Please see Supplemental Table 2 for standardized dose estimates for the reference adult for ${ }^{177} \mathrm{Lu}$-DOTATATE (20). Although ${ }^{68} \mathrm{Ga}$-DOTATATE may be used for diagnostic evaluation of tracer uptake, it cannot be used for dosimetry planning due to its short (68 min) half-life. However, ${ }^{177} \mathrm{Lu} \gamma$-rays are suitable for $\gamma$-camera imaging (Supplemental Table 3), and imaging for dosimetry may be performed after the initial therapy cycle (Fig. 4). Further details about potential imaging protocols for dosimetry can be found in the supplemental materials.

\section{TOXICITY}

Rates of toxicity vary between patients, and heavily pretreated patients have higher rates of PRRT-associated toxicity. In particular, liver toxicity has been reported in patients who have had extensive prior liver-directed therapy or who are treated with ${ }^{90} \mathrm{Y}-$ based PRRT (21). The following sections focus on bone marrow and kidney toxicity in more detail.

\section{Bone Marrow Toxicity}

Grade 3 and 4 thrombocytopenia and neutropenia occur in $\leq 5 \%$ of patients and resolve within 8 wk $(4,22)$. Grade 3 and 4 lymphopenia is more common but is rarely of clinical significance since opportunistic infections are not observed in association with ${ }^{177} \mathrm{Lu}$-DOTATATE. The most significant long-term hematologic risk is myelodysplastic syndrome or acute leukemia, which occurs in roughly $2 \%-3 \%$ of patients at a median of $2 \mathrm{y}$ after therapy. Although little can be done during treatment to minimize marrow toxicity, there are possible risk factors for the development of toxicity such as prior chemotherapy $(6,23,24)$.

\section{Renal Toxicity}

Because of the high exposure to the kidneys from the renal excretion of the radiotracer, renal toxicity is possible. With the introduction of concurrent amino acids for renal protection and the use ${ }^{177} \mathrm{Lu}$-labeled compounds in place of ${ }^{90}$ Y-labeled compounds, the rates of renal toxicity are low, with long-term grade 3-4 renal toxicity less than $2 \%(4,23)$. Variations in individual kidney sensitivity to radiation may explain the variability seen between renal dose and the subsequent development of renal toxicity. For example, there is evidence that patients with long-standing diabetes or hypertension may be at higher risk for renal dysfunction after ${ }^{177} \mathrm{Lu}-$ DOTATATE treatment (19). Nevertheless, severe renal toxicity is rare $(<5 \%)$ when using the current administration guidelines $(4,19)$.

\section{FOLLOW-UP}

Monitoring of patients after PRRT treatment is an essential part of the treatment plan. The recommended monitoring should include clinical evaluation to assess symptoms and detection of possible treatment sequelae, laboratory, and imaging tests (Table 5).

\section{Clinical Evaluation}

Clinical evaluation by the treating or primary team should ideally be conducted at $1 \mathrm{mo}, 3 \mathrm{mo}, 6 \mathrm{mo}$, and $12 \mathrm{mo}$ after PRRT. If there are no laboratory abnormalities or clinical symptoms concerning for posttreatment sequelae, patients can resume clinical follow-up per the primary team. National Comprehensive Cancer Network (NCCN) guidelines recommend follow-up intervals of 312 mo based on clinical presentation (25). Clinical symptoms and presentations that could reflect possible progression, increased symptoms from carcinoid syndrome, or posttreatment sequelae warrant closer monitoring.

\section{Laboratory Tests and Markers}

Blood tests including complete blood count with differential, aspartate aminotransferase, alanine aminotransferase, alkaline phosphatase, total bilirubin, albumin, and serum creatinine/GFR should

TABLE 5

Recommended Monitoring Interval After Completion of PRRT

\begin{tabular}{|c|c|c|c|c|}
\hline Time after treatment* & Clinical evaluation & Laboratory tests ${ }^{\dagger}$ & Markers ${ }^{\ddagger}$ & Diagnostic imaging \\
\hline $2-4 w k$ & $X$ & $X$ & & \\
\hline $2 \mathrm{mo}$ & & $X$ & & XI $\}$ \\
\hline $3 \mathrm{mo}$ & $X$ & $X$ & Per team & \\
\hline $6 \mathrm{mo}$ & $X$ & $X$ & Per team & $X$ \\
\hline $12 \mathrm{mo}$ & $x$ & $X$ & Per team & $x$ \\
\hline Long term & Per team & Per team & Per team & Per team \\
\hline \multicolumn{5}{|c|}{$\begin{array}{l}{ }^{*} \text { Increase monitoring based on clinical presentation, symptoms, concern for progressive disease, or posttreatment sequelae. } \\
{ }^{\dagger} \text { Complete blood count with differential, aspartate aminotransferase, alanine aminotransferase, alkaline phosphatase, total bilirubin, } \\
\text { bumin, and serum creatinine/GFR } \\
{ }^{\ddagger} \text { Monitoring of markers should be based on clinical indication/presentation. } \\
\text { IImaging is recommended once between one and three months after therapy. }\end{array}$} \\
\hline
\end{tabular}


be monitored at roughly $1 \mathrm{mo}, 3 \mathrm{mo}, 6 \mathrm{mo}$, and $12 \mathrm{mo}$ after treatment. If all blood tests are within normal limits, a complete blood count and serum creatinine should be monitored at least yearly or sooner if clinically indicated or per primary team. If there are any abnormalities in blood tests, more frequent monitoring is recommended.

Both secondary myelodysplastic syndrome and acute leukemia are known toxicities of PRRT. Increased monitoring is recommended for those patients with persistent cytopenias with blood tests until recovery. In addition, hematology consult should be considered for patients with persistent cytopenias.

Patients with mild to moderate renal impairment after PRRT treatment warrant closer monitoring with serum creatinine measurements. In addition, nephrology consult should be considered for patients with persistent or worsening renal impairment.

There are no formal recommendations for following tumor markers after PRRT. NCCN NET guidelines recommend following markers if clinically applicable every 3-12 mo (25).

\section{Diagnostic Imaging Evaluation}

In most cases, diagnostic imaging should be done at 1-3 mo, 6 mo, and 12 mo after the completion of all treatment cycles. Thereafter, patients should undergo diagnostic imaging based on treatment response. It is important to remain consistent with imaging modalities. NCCN guidelines recommend following patients with contrast-enhanced abdominal and pelvic CT or MRI with contrast along with chest CT with or without contrast (if clinically indicated) every 3-12 mo (25). MRI of the liver with gadoxetate disodium (Eovist) should be considered for those patients with liver-dominant disease $(26,27)$. Increased monitoring is recommended for clinical presentation concerning for possible progressive disease, clinical worsening, or possible posttreatment sequel. If there is evidence of progression or equivocal findings on CT or MRI, SSTR-based imaging with ${ }^{68} \mathrm{Ga}$-DOTATATE PET should be considered and is preferred over SSTR scintigraphy ( ${ }^{111} \mathrm{In}-$ pentetreotide) (2).

It should be noted that the first imaging study performed after the completion of PRRT can be complicated by pseudoprogression (28). In nearly $10 \%$ of patients with stable disease, metastatic disease can increase in volume transiently, presumably due to edema from radiation to the tumor. Diagnostic imaging during the course of PRRT can be considered in patients with relatively aggressive tumors, or patients with clinical evidence of progression.

\section{CONCLUSION}

${ }^{177} \mathrm{Lu}$-DOTATATE is an effective treatment for patients with NETs. The above guidelines provide information on how to safely administer this novel treatment to patients.

\section{REFERENCES}

1. Kulke MH, Mayer RJ. Carcinoid tumors. N Engl J Med. 1999;340:858-868.

2. Hope TA, Bergsland EK, Bozkurt MF, et al. Appropriate use criteria for somatostatin receptor PET imaging in neuroendocrine tumors. $J$ Nucl Med. 2018;59: 66-74.

3. Kwekkeboom DJ, Kam BL, van Essen M, et al. Somatostatin-receptor-based imaging and therapy of gastroenteropancreatic neuroendocrine tumors. Endocr Relat Cancer. 2010;17:R53-R73.

4. Strosberg J, El-Haddad G, Wolin E, et al. Phase 3 trial of ${ }^{177}$ Lu-Dotatate for midgut neuroendocrine tumors. N Engl J Med. 2017;376:125-135.

5. Kwekkeboom DJ, de Herder WW, Kam BL, et al. Treatment with the radiolabeled somatostatin analog [177 Lu-DOTA 0,Tyr3]octreotate: toxicity, efficacy, and survival. J Clin Oncol. 2008;26:2124-2130.
6. Lutathera [package insert]. New York, NY: Advanced Accelerator Applications; 2018.

7. de Jong M, Krenning E. New advances in peptide receptor radionuclide therapy. J Nucl Med. 2002;43:617-620.

8. Bodei L, Mueller-Brand J, Baum RP, et al. The joint IAEA, EANM, and SNMMI practical guidance on peptide receptor radionuclide therapy (PRRNT) in neuroendocrine tumours. Eur J Nucl Med Mol Imaging. 2013;40: 800-816.

9. Roscoe JA, Morrow GR, Aapro MS, Molassiotis A, Olver I. Anticipatory nausea and vomiting. Support Care Cancer. 2011;19:1533-1538.

10. Abbott A, Sakellis C, Andersen E. Nuts and bolts of ${ }^{177}$ Lu-DOTATATE administration in the nuclear medicine division: guidance from a single institute's experience. J Nucl Med Technol. August 3, 2018 [Epub ahead of print].

11. de Keizer B, van Aken MO, Feelders RA, et al. Hormonal crises following receptor radionuclide therapy with the radiolabeled somatostatin analogue $\left[{ }^{177} \mathrm{Lu}-\right.$ DOTA0,Tyr3]octreotate. Eur J Nucl Med Mol Imaging. 2008;35:749-755.

12. Tapia Rico G, Li M, Pavlakis N, Cehic G, Price TJ. Prevention and management of carcinoid crises in patients with high-risk neuroendocrine tumours undergoing peptide receptor radionuclide therapy (PRRT): literature review and case series from two Australian tertiary medical institutions. Cancer Treat Rev. 2018;66: $1-6$.

13. Bonta DV, Halkar RK, Alazraki N. Extravasation of a therapeutic dose of ${ }^{131} \mathrm{I}-$ metaiodobenzylguanidine: prevention, dosimetry, and mitigation. J Nucl Med. 2011;52:1418-1422.

14. Siegel JA, Marcus CS, Stabin MG. Licensee over-reliance on conservatisms in NRC guidance regarding the release of patients treated with ${ }^{131}$ I. Health Phys. 2007;93:667-677.

15. Regulatory Guide 8.39: Release of Patients Administered Radioactive Materials. U.S. Nuclear Regulatory Commission website. https://www.nrc.gov/docs/ML0833/ ML083300045.pdf. Accessed February 21, 2019.

16. Stabin MG. RADAR patient exposure radiation dose calculator. RADAR website. http://www.doseinfo-radar.com/ExposureCalculator.html. Accessed February $21,2019$.

17. National Council on Radiation Protection and Measurements. Management of Persons Contaminated with Radionuclides. Bethesda, MD: National Council on Radiation; 2008.

18. Cremonesi M, Botta F, Di Dia A, et al. Dosimetry for treatment with radiolabelled somatostatin analogues: a review. Q J Nucl Med Mol Imaging. 2010;54: 37-51.

19. Bodei L, Cremonesi M, Grana CM, et al. Peptide receptor radionuclide therapy with ${ }^{177} \mathrm{Lu}$-DOTATATE: the IEO phase I-II study. Eur J Nucl Med Mol Imaging. 2011;38:2125-2135.

20. Sandström M, Garske-Román U, Granberg D, et al. Individualized dosimetry of kidney and bone marrow in patients undergoing ${ }^{177} \mathrm{Lu}$-DOTA-octreotate treatment. J Nucl Med. 2013;54:33-41.

21. Riff BP, Yang Y-X, Soulen MC, et al. Peptide receptor radionuclide therapyinduced hepatotoxicity in patients with metastatic neuroendocrine tumors. Clin Nucl Med. 2015;40:845-850.

22. Brabander T, van der Zwan WA, Teunissen JJM, et al. Long-term efficacy, survival, and safety of $\left[{ }^{177} \operatorname{Lu}-\operatorname{DOTA}(0), \operatorname{Tyr}(3)\right]$ octreotate in patients with gastroenteropancreatic and bronchial neuroendocrine tumors. Clin Cancer Res. 2017;23: 4617-4624.

23. Bodei L, Kidd M, Paganelli G, et al. Long-term tolerability of PRRT in 807 patients with neuroendocrine tumours: the value and limitations of clinical factors. Eur J Nucl Med Mol Imaging. 2015;42:5-19.

24. Bergsma H, van Lom K, Raaijmakers MHGP, et al. Persistent hematologic dysfunction after peptide receptor radionuclide therapy with ${ }^{177}$ Lu-DOTATATE: incidence, course, and predicting factors in patients with gastroenteropancreatic neuroendocrine tumors. J Nucl Med. 2018;59:452-458.

25. Kulke MH, Shah MH, Benson AB, et al. Neuroendocrine tumors, version 1.2015. J Natl Compr Canc Netw. 2015;13:78-108.

26. Tirumani SH, Jagannathan JP, Braschi-Amirfarzan M, et al. Value of hepatocellular phase imaging after intravenous gadoxetate disodium for assessing hepatic metastases from gastroenteropancreatic neuroendocrine tumors: comparison with other MRI pulse sequences and with extracellular agent. Abdom Radiol. 2018;43: 2329-2339.

27. Morse B, Jeong D, Thomas K, Diallo D, Strosberg JR. Magnetic resonance imaging of neuroendocrine tumor hepatic metastases: does hepatobiliary phase imaging improve lesion conspicuity and interobserver agreement of lesion measurements? Pancreas. 2017;46:1219-1224.

28. Brabander T, van der Zwan WA, Teunissen JJM, et al. Pitfalls in the response evaluation after peptide receptor radionuclide therapy with $\left[{ }^{177} \mathrm{Lu}\right.$-DOTA0,Tyr3] octreotate. Endocr Relat Cancer.2017;24:243-251. 